Yuji Sano*

\title{
A polar dual to the momentum of toric Fano manifolds
}

https://doi.org/10.1515/coma-2020-0116

Received September 18, 2020; accepted July 5, 2021

Abstract: We introduce an invariant on the Fano polytope of a toric Fano manifold as a polar dual counterpart to the momentum of its polar dual polytope. Moreover, we prove that if the momentum of the polar dual polytope is equal to zero, then the dual invariant on a Fano polytope vanishes.

Keywords: Kähler-Einstein metrics, toric Fano manifolds, Futaki invariant, Fano polytopes

MSC: 14M25; 14J45; 32Q20

\section{Introduction}

For a given smooth toric Fano manifold $X$, let $P$ be a so-called Fano polytope induced from the complete fan of $X$. Let $P^{\star}$ be the polar dual polytope to $P$, which is the image of $X$ under the moment map. The purpose of this note is to propose an invariant on $P$ which is a polar dual counterpart to the momentum of $P^{\star}$.

Our motivation comes from the existence problem of Kähler-Einstein metrics on toric Fano manifolds. Futaki introduced the integral invariant (so-called Futaki invariant) in [5] as an obstruction to the existence of Kähler-Einstein metrics. Wang and Zhu in [16] showed that any toric Fano manifold $X$ admits Kähler-Einstein metrics if Futaki invariant vanishes. Mabuchi showed in [9] that Futaki invariant on the toric Fano manifold $X$ corresponds to the momentum of $P^{\star}$. These results induce that if the momentum of $P^{\star}$ is equal to the origin, then $X$ admits a Kähler-Einstein metrics. Our motivation is to give an equivalent condition on not $P^{\star}$ but $P$ to the vanishing of Futaki invariant on toric Fano manifolds. If we consider this problem in the context of complex differential geometry, the answer would be the vanishing of some canonical vector fields associated with such as Kähler-Ricci solitons ([15]) and extremal Kähler metrics ([6]). However, such vector fields seem to be relevant to geometry of $P^{\star}$ rather than $P$. For example, see the computation of the extremal vector fields by Nakagawa [10].

To state our statement, let us fix the notations. Let $N \simeq \mathbb{Z}^{n}$ be a lattice of rank $n$ and let $M$ be its dual lattice $\operatorname{Hom}_{\mathbb{Z}}(N, \mathbb{Z})$. Set $N_{\mathbb{R}}:=N \otimes_{\mathbb{Z}} \mathbb{R}$ and $M_{\mathbb{R}}:=M \otimes_{\mathbb{Z}} \mathbb{R}$. For $\mathbf{x} \in N_{\mathbb{R}}$ and $\mathbf{y} \in M_{\mathbb{R}}$, we denote by $\langle\mathbf{x}, \mathbf{y}\rangle$ the standard pairing.

We call a lattice polytope $P \subset N_{\mathbb{R}}$ of dimension $n$ a Fano polytope if $P$ satisfies the following conditions:

(a) the origin is contained in the interior of $P$,

(b) any face of $P$ is a simplex, and

(c) the set of vertices of any facet of $P$ constitutes a basis of $N$.

Let

$$
\mathrm{V}(P):=\left\{\mathbf{u}_{i} \in N \mid 1 \leq i \leq K\right\}
$$

^Corresponding Author: Yuji Sano: Department of Applied Mathematics, Faculty of Sciences, Fukuoka University, 8-19-1 Nanakuma, Jonan-ku Fukuoka 814-0180, Japan, E-mail: sanoyuji@fukuoka-u.ac.jp 
be the set of vertices of $P$. The polar dual of $P$ is defined by

$$
P^{\star}:=\left\{\mathbf{y} \in M_{\mathbb{R}} \mid\left\langle\mathbf{u}_{i}, \mathbf{y}\right\rangle \geq-1 \text { for all } \mathbf{u}_{i} \in \mathrm{V}(P)\right\},
$$

which is also a convex lattice polytope in $M_{\mathbb{R}}$. A vertex $\mathbf{u}_{i}$ of $P$ is the normal of some facet $F_{i}$ of $P^{\star}$. Let

$$
\mathrm{V}\left(P^{\star}\right):=\left\{\mathbf{v}_{j} \in N \mid 1 \leq j \leq L\right\}
$$

be the set of vertices of $P^{\star}$. By definition (c) of $P$, each vertex $\mathbf{v}_{j}$ meets precisely $n$ edges, more precisely, $\mathbf{v}_{j}$ is the apex of an $n$-dimensional cone with $n$ edges whose primitive vectors

$$
\left\{\mathbf{e}_{j, k}^{\star} \in M \mid 1 \leq k \leq n\right\}
$$

constitutes a basis of $M$. We denote the dual basis to $\left\{\mathbf{e}_{j, k}^{\star}\right\}$ by $\left\{\mathbf{e}_{j, k}\right\}$. The set $\left\{\mathbf{e}_{j, k}\right\}$ is equal to the set of the vertices of some facet of $P$.

Let us consider the following sum of rational functions on $N_{\mathbb{R}}$

$$
\mathbf{m}_{P^{\star}}:=\sum_{j=1}^{L} \frac{\mathbf{v}_{j}^{n+1}}{\prod_{k=1}^{n} \mathbf{e}_{j, k}^{\star}} .
$$

Through this article, we often identify an element in $M_{\mathbb{R}}$ with a polynomial on $N_{\mathbb{R}}$ of degree one, and vice versa. In the contexts of the theory of equivariant cohomology on toric varieties, it is known that a sum of rational function such as $\mathbf{m}_{P^{*}}$ is a homogeneous polynomial of degree one (cf. Proposition 1.1 in [8], also see Corollary 4). Moreover, by a consequence of the theory of counting lattice points in polytopes (also the localization of equivariant cohomology), we can see that $\mathbf{m}_{P^{\star}}$ is equal to the momentum of $P^{\star}$ up to the multiplication of constant, i.e.,

$$
\mathbf{m}_{P^{\star}}=C \int_{P^{*}} \mathbf{y} d \mathbf{y}
$$

for some non-zero constant $C$ (see Proposition 7). Here $d \mathbf{y}$ denotes the standard Lebesgue measure on $M_{\mathbb{R}} \simeq$ $\mathbb{R}^{n}$. We will see these facts in the next section in detail.

Next, let us consider the following vector in $N_{\mathbb{R}}$ (equivalently, the linear function on $M_{\mathbb{R}}$ )

$$
\mathbf{e}_{P}:=\sum_{i=1}^{K} \sharp \mathrm{V}\left(F_{i}\right) \cdot \mathbf{u}_{i}=\sum_{j=1}^{L}\left(\sum_{k=1}^{n} \mathbf{e}_{j, k}\right) .
$$

Here we denote by $\sharp \mathrm{V}\left(F_{i}\right)$ the number of vertices of the corresponding facet $F_{i}$ of $P^{\star}$ to a vertex $\mathbf{u}_{i}$ of $P$.

Now, we will see a relation between $\mathbf{e}_{P}$ and $\mathbf{m}_{P^{\star}}$ as follows. By definition of $P$, the origin is the only lattice point in the interior of $P^{\star}$. Then, we have

$$
\mathbf{v}_{j}=-\sum_{k=1}^{n} \mathbf{e}_{j, k}^{\star},
$$

otherwise $\mathbf{v}_{j}+\sum_{k=1}^{n} \mathbf{e}_{j, k}^{\star}$ would be the lattice point in the interior of $P^{\star}$ except the origin. Substituting (3) into (1), we can rewrite $\mathbf{m}_{P^{\star}}$ by

$$
\mathbf{m}_{P^{\star}}=-\sum_{j=1}^{L} \frac{\mathbf{v}_{j}^{n}}{\prod_{k=1}^{n} \mathbf{e}_{j, k}^{\star}}\left(\sum_{k=1}^{n} \mathbf{e}_{j, k}^{\star}\right) .
$$

Comparing (4) with (2) in each $j$, we can see that $\mathbf{m}_{P^{\star}}$ is polar dual to $\mathbf{e}_{P}$ by regarding

$$
-\frac{\mathbf{v}_{j}^{n}}{\prod_{k=1}^{n} \mathbf{e}_{j, k}^{\star}}
$$

as the coefficient in (4) (See also (15)).

The main result of this note is as follows.

Theorem 1. Let $P$ be an n-dimensional Fano polytope. If $\mathbf{m}_{P^{\star}}=\mathbf{o}$, then $\mathbf{e}_{P}=\mathbf{o}$. 
This theorem means that the symmetry of $P$ is an obstruction to the existence of Kähler-Einstein metrics on the associated smooth Fano manifold $X$. An advantage of $\mathbf{e}_{P}$ over $\mathbf{m}_{P^{\star}}$ is the facility of computation. In fact, we can compute $\mathbf{e}_{P}$ only by counting the number of vertices of the facets of $P^{\star}$ (or equivalently the number of the edges from the vertices of $P$ ). On the other hand, there are counterexamples to the the converse of Theorem 1 (see Section 4).

We finish this section with two remarks. First, the statement of Theorem 1 follows immediately if $P$ is symmetric in the sense of [1], i.e., the fixed point under the Weyl group $\mathcal{W}(P) \subset G L(n, \mathbb{Z})\left(\right.$ resp. $\left.\mathcal{W}\left(P^{\star}\right)\right)$ preserving $P \subset N_{\mathbb{R}}$ (resp. $P^{\star}$ ) is only the origin. If $P$ is symmetric, then both of $\mathbf{m}_{P^{*}}$ and $\mathbf{e}_{P}$ vanish, because they are unchanged under $\mathcal{W}\left(P^{\star}\right)$ and $\mathcal{W}(P)$ respectively. With the classification, Fano polytopes whose $\mathbf{m}_{P^{\star}}$ vanishes are symmetric up to dimension 6 . Then, $\mathbf{e}_{P}$ also vanishes. As interesting examples found in [13], which are non-symmetric Fano polytopes with $\mathbf{m}_{P^{*}}=\mathbf{o}$. The statement of Theorem 1 is true for such examples. Secondly, by definition, $\mathbf{e}_{P}$ coincides with the momentum $\mathbf{m}_{P}$ of $P$ modulo the multiplication of constant. However, we cannot replace $\mathbf{e}_{P}$ by $\mathbf{m}_{P}$ in Theorem 1 if $X_{P^{\star}}$ is singular. In fact, there exists a counterexample (Remark 5.3 [11]) with $\mathbf{m}_{P^{*}}=\mathbf{o}$ and $\mathbf{m}_{P} \neq \mathbf{o}$ if $P$ is reflexive (not smooth).

The organization of this note is as follows. In Section 2, we collect the facts needed for the proof of our main theorem from the theory of piecewise polynomials. In particular, we see that $\mathbf{m}_{P^{\star}}$ is the momentum of $P^{\star}$. In Section 3, we observe some combinatorial structure of $P^{\star}$ with $\mathbf{m}_{P^{\star}}=\mathbf{o}$. With this, we complete the proof. In Section 4, we introduce some counterexamples to the converse of Theorem 1.

\section{Invariants via piecewise polynomials}

To prove Theorem 1, we make use of the theory of piecewise polynomials on polytopes. We refer to [8] for the terminologies and facts in this section.

Let us fix notations. Let $P$ be an $n$-dimensional Fano polytope and $\Sigma(P)$ be the associated complete fan, that is,

$$
\Sigma(P):=\{\mathbf{o}\} \cup\{\operatorname{cone}(G)\}_{G: \text { face of } P}
$$

where cone $(G)$ is the cone over $G$. Take a maximal cone $\sigma_{j} \in \Sigma(P)(1 \leq j \leq L)$. It corresponds to some vertex $\mathbf{v}_{j}$ of $P^{\star}$. The cone $\sigma_{j}$ is spanned by a basis $\left\{\mathbf{e}_{j, k} \mid 1 \leq k \leq n\right\}$ of $N$. Let $\left\{\mathbf{e}_{j, k}^{\star} \mid 1 \leq k \leq n\right\}$ be its dual basis.

For each $\sigma$, we define the equivariant multiplicities (of the nondegenerate torus fixed point of $X_{P^{\star}}$ corresponding to $\sigma$ ) by

$$
e_{\sigma_{j}}:=\frac{1}{\prod_{k=1}^{n} \mathbf{e}_{j, k}^{\star}}
$$

which is a homogeneous rational function of degree $-n$ over $N_{\mathbb{R}}$. For a subcone $\tau$ in $\sigma$, we define

$$
e_{\tau, \sigma}:=\frac{1}{\prod_{\mathbf{e}_{j, k} \notin \tau} \mathbf{e}_{j, k}^{\star}} .
$$

A crucial feature of $e_{\tau, \sigma}$ is

Proposition 2 (Lemma $3.2[8]$ ).

$$
\sum_{\tau \preceq \sigma} e_{\tau, \sigma}=\left\{\begin{array}{l}
0 \text { for } \operatorname{dim} \tau<n, \\
1 \text { for } \operatorname{dim} \tau=n .
\end{array}\right.
$$

Let $F$ be an integral piecewise polynomial on $\Sigma(P)$, i.e., a continuous function $F: N_{\mathbb{R}} \rightarrow \mathbb{R}$ such that the restriction $\left.F\right|_{\sigma}$ of $F$ to each maximal cone $\sigma$ is a polynomial with integral coefficients. We denote by $P P^{\bullet}(\Sigma(P))$ the ring of integral piecewise polynomials. For each ray $\mathbb{R} \cdot \mathbf{u}_{i}$ in $\Sigma(P)$, we associate a unique integral piecewise linear function $\Psi_{i}$ satisfying $\Psi_{i}\left(\mathbf{u}_{j}\right)=\delta_{i j}$ for all ray $\mathbb{R} \cdot \mathbf{u}_{j}$ in $\Sigma(P)$. For any cone $\tau$ in $\Sigma(P)$, we associate

$$
\Psi_{\tau}:=\prod_{\mathbf{u}_{i} \in \tau} \Psi_{i},
$$


which vanishes away from the union of the cones in $\Sigma(P)$ that contains $\tau$. Since $P P^{\bullet}(\Sigma(P))$ is generated by $\left\{\Psi_{\tau}\right\}_{\tau \in \Sigma(P)}$ as a $\operatorname{Sym}^{\bullet}(M)$-module, the equality (5) implies the following result.

Proposition 3 (Proposition 1.1 [8]). Let $F \in P P^{k}(\Sigma(P))$ be an integral piecewise polynomial of degree $k$. Then,

$$
\left.\sum_{\sigma: \text { maximal }} e_{\sigma} F\right|_{\sigma}
$$

is a homogeneous polynomial of degree $k-n$. In particular, if $k<n$ then $\left.\sum e_{\sigma} F\right|_{\sigma}$ vanishes. If $k=n$, then $\left.\sum e_{\sigma} F\right|_{\sigma}$ is an integer.

Corollary 4. The invariant $\mathbf{m}_{P^{\star}}$ is a linear function on $N_{\mathbb{R}}$.

Proof. Let

$$
F_{b}=(-1)^{n+1}\left(\sum_{i=1}^{K} \Psi_{i}\right)^{n+1}
$$

By definition of $\Psi_{i}$ and (3), we find

$$
\mathbf{m}_{P^{\star}}=\left.\sum_{\sigma: \text { maximal }} e_{\sigma} F_{b}\right|_{\sigma}
$$

Proposition 3 implies the statement to be proved. The proof is completed.

Next we will see that $\mathbf{m}_{P^{\star}}$ is equal to the momentum of $P^{\star}$ (modulo the multiplication of constant) following Section 5 in [8]. For each vertex $\mathbf{v}_{j}$ of $P^{\star}$, let consider the cone $C_{\mathbf{v}_{j}}^{\star}$ in $M_{\mathbb{R}}$, whose apex is the origin, given by

$$
\mathcal{C}_{\mathbf{v}_{j}}^{\star}=\mathbb{R}_{\geq 0} \cdot \mathbf{e}_{j, 1}^{\star}+\cdots+\mathbb{R}_{\geq 0} \cdot \mathbf{e}_{j, n}^{\star}
$$

where $\left\{\mathbf{e}_{j, k}^{\star}\right\}$ is the set of primitive vectors of edges of $P^{\star}$ from $\mathbf{v}_{j}$. Let

$$
\operatorname{Hilb}\left(\mathcal{C}_{\mathbf{v}_{j}}^{\star}\right)(\mathbf{x})=\sum_{\mathbf{y} \in \mathcal{C}_{\mathbf{v}_{j} \cap M}^{\star}} \mathbf{x}^{\mathbf{y}},
$$

where $\mathbf{x}^{\mathbf{y}}=\prod_{i=1}^{n} x_{i}^{y_{i}}$. This is so-called the multigraded Hilbert series of the cone $\mathcal{C}_{\mathbf{v}_{j}}^{\star}$. Since $\operatorname{Hilb}\left(\mathcal{C}_{\mathbf{v}_{j}}^{\star}\right)$ is a generating function for the lattice points contained in the unimodular cone $\mathcal{C}_{\mathbf{v}_{j}}^{\star}$ spanned by $\left\{\mathbf{e}_{j, k}^{\star}\right\}_{1 \leq k \leq n}$, we have (cf. Theorem 3.5 [2])

$$
\operatorname{Hilb}\left(\mathcal{C}_{\mathbf{v}_{j}}^{*}\right)(\mathbf{x})=\frac{1}{\prod_{k=1}^{n}\left(1-\mathbf{x}_{j, k}^{\mathbf{e}_{j, k}}\right)} .
$$

First, we recall the following well-known result with the proof.

Proposition 5 (Proposition 5.2 [8]). The volume of $P^{\star}$ is equal to

$$
\operatorname{vol}_{n}\left(P^{\star}\right)=\frac{(-1)^{n}}{n !} \sum_{j=1}^{L} \frac{\mathbf{v}_{j}^{n}}{\prod_{k=1}^{n} \mathbf{e}_{j, k}^{\star}} .
$$

Proof. The volume of $P^{\star}$ is reduced to the following lattice point counting

$$
\begin{aligned}
\operatorname{vol}_{n}\left(P^{\star}\right) & =\operatorname{vol}_{n}\left(P^{\star}, \ldots, P^{\star}\right) \\
& \left.=\frac{1}{n !} \sum_{l=1}^{n}(-1)^{n-l}\left(\begin{array}{c}
n \\
l
\end{array}\right) \sharp(\underbrace{P^{\star}+\cdots+P^{\star}}_{l}) \cap M\right) .
\end{aligned}
$$


Here $\operatorname{vol}_{n}\left(P^{\star}, \ldots, P^{\star}\right)$ and $P^{\star}+\cdots+P^{\star}$ denote the mixed volume and the Minkowski sum of $\left(P^{\star}, \ldots, P^{\star}\right)$ respectively. The right hand of (7) is equal to the principal part of

$$
\frac{1}{n !} \sum_{l=1}^{n}(-1)^{n-l}\left(\begin{array}{c}
n \\
l
\end{array}\right) \sum_{\mathbf{y} \in(\underbrace{P^{\star}+\cdots+P^{\star}}_{l}) \cap M} \mathbf{x}^{\mathbf{y}}
$$

at $\mathbf{x}=\mathbf{1}_{T}:=(1, \ldots, 1)$. In the above, we use the fact that the principal part of $\mathbf{x}^{\mathbf{y}}$ at $\mathbf{1}_{T}$ is equal to one.

Brion's formula [3] implies that (8) is equal to

$$
\frac{1}{n !} \sum_{j=1}^{L}\left(\mathbf{x}^{\mathbf{v}_{j}}-1\right)^{n} \operatorname{Hilb}\left(\mathcal{e}_{\mathbf{v}_{j}}^{\star}\right)(\mathbf{x})
$$

by expanding $\left(\mathbf{x}^{\mathbf{v}_{j}}-1\right)^{n}$.

Therefore, we get the desired equality, because the principal parts of $\mathbf{x}^{\mathbf{v}_{j}}-1$ and $1-\mathbf{x}^{\mathbf{e}_{j, k}^{*}}$ at $\mathbf{1}_{T}$ are $\mathbf{v}_{j}$ and $-\mathbf{e}_{j, k}^{\star}$ respectively and the principal parts are multiplicative.

For a facet $F$ of $P^{\star}$, we introduce the relative volume $A(F)$ by

$$
A(F)=\int_{F} d \mu
$$

where $d \mu$ is the measure on the facet

$$
F=\left\{\mathbf{y} \in M_{\mathbb{R}} \mid h(\mathbf{y})=\langle\mathbf{u}, \mathbf{y}\rangle-c=0\right\}
$$

defined by

$$
d h \wedge d \mu=d \mathbf{y}
$$

up to sign. In the above, the polynomial $h$ is the defining polynomial of $F$ and $\mathbf{u}$ is the primitive normal vector of $F$. Remark that $A(F)$ is equal to the lattice volume of $F$, which is measured so that the volume of the fundamental paralloped in the minimal sublattice in $M_{\mathbb{Z}}$ containing the vertices of $F$. Then, a similar proof as (6) for the sublattice containing the vertices of $F$ works as follows.

Proposition 6. The relative volume of $F$ is equal to

$$
A(F)=\frac{(-1)^{n-1}}{(n-1) !} \sum_{\mathbf{v} \in \mathrm{V}(F)} \frac{\mathbf{v}_{j}^{n-1}}{\prod_{k=1}^{n-1} \mathbf{e}_{j, k}^{\star}} .
$$

Here the summation runs through all the vertices $\mathbf{v}$ of $F$ and $\left\{\mathbf{e}_{j, k}^{\star}\right\}_{k=1, \cdots, n-1}$ are the set of edge vectors from $\mathbf{v}_{j}$ parallel to $F$.

Considering the first variation of (6), we find the following result.

Proposition 7. The invariant $\mathbf{m}_{P^{\star}}$ is equal to the momentum of $P^{\star}$ modulo the multiplication of constant.

Proof. The momentum of $P^{\star}$

$$
\int_{P^{\star}} \mathbf{y} d \mathbf{y}
$$

is equal (up to constant) to the principal part of

$$
\frac{1}{n !} \sum_{l=1}^{n}(-1)^{n-l}\left(\begin{array}{c}
n \\
l
\end{array}\right) \sum_{\mathbf{y} \in \underbrace{P^{\star}+\cdots+P^{\star}}_{l} \cap M M}\left(\mathbf{x}^{\mathbf{y}}-1\right)
$$


at $\mathbf{1}_{T}$, because the principal part of $\mathbf{x}^{\mathbf{y}}-1$ is equal to $\mathbf{y}$.

On the other hand, (10) is equal to

$$
\frac{1}{n !} \sum_{j=1}^{L}\left(\mathbf{x}^{\mathbf{v}_{j}}-1\right)^{n} \operatorname{Hilb}\left(\mathcal{C}_{\mathbf{v}_{j}}^{\star}\right)(\mathbf{x})-\frac{(-1)^{n}}{n !} \sum_{j=1}^{L} \frac{\mathbf{v}_{j}^{n}}{\prod_{k=1}^{n} \mathbf{e}_{j, k}^{\star}} .
$$

Then, by using (3), the principal part of (11) at $\mathbf{1}_{T}$ is equal to

$$
\begin{aligned}
\frac{(-1)^{n}}{n !}\left\{\sum_{j=1}^{L} \frac{n \mathbf{v}_{j}^{n+1}}{2 \prod_{k=1}^{n} \mathbf{e}_{j, k}^{\star}}+\sum_{j=1}^{L} \mathbf{v}_{j}^{n} \frac{\sum_{k=1}^{n}\left(-\mathbf{e}_{j, k}^{\star}\right)}{\prod_{k=1}^{n} \mathbf{e}_{j, k}^{\star}}\right\} & =\frac{(-1)^{n}\left(\frac{1}{2} n+1\right)}{n !} \sum_{j=1}^{L} \frac{\mathbf{v}_{j}^{n+1}}{\prod_{k=1}^{n} \mathbf{e}_{j, k}^{\star}} \\
& =\frac{(-1)^{n}\left(\frac{1}{2} n+1\right)}{n !} \mathbf{m}_{P^{\star}} .
\end{aligned}
$$

In the above, we use the following calculations: the principal parts of $\mathbf{x}^{\mathbf{v}_{j}}-1-\mathbf{v}_{j}\left(\operatorname{resp} \cdot \frac{1}{1-\mathbf{x}^{\mathbf{e}_{j, k}^{+}}}\right.$at $\left.\mathbf{1}_{T}\right)$ is equal to $\frac{1}{2} \mathbf{v}_{j}^{2}$ (resp. 1). The proof is completed.

We introduce the relative momentum of the facet $F_{i}$ by

$$
\mathbf{m}\left(F_{i}\right):=\sum_{\mathbf{v}_{j} \in \mathrm{V}\left(F_{i}\right)} \frac{\mathbf{v}_{j}^{n} \mathbf{e}_{j, k_{i}}^{\star}}{\prod_{k=1}^{n} \mathbf{e}_{j, k}^{\star}} .
$$

Proposition 3 implies that $\mathbf{m}\left(F_{i}\right)$ is a linear function, i.e., $\mathbf{m}\left(F_{i}\right) \in M_{\mathbb{Z}}$. With the same proof as Proposition 7 , we can see the following proposition.

Proposition 8. For any facet $F_{i}$ of $P^{\star}$, its relative momentum $\mathbf{m}\left(F_{i}\right)$ is equal to

$$
C \int_{F_{i}} \mathbf{y} d \mu_{i}
$$

for some positive constant $C$ independent of $i$. Here the measure $d \mu_{i}$ is defined in (9).

To prove Theorem 1, we introduce another invariant on $P$ as follows:

$$
\widetilde{\mathbf{e}}_{P}:=\sum_{i=1}^{K} \sum_{\mathbf{v}_{j} \in \mathrm{V}\left(F_{i}\right)} \frac{\mathbf{v}_{j}^{n-1} \mathbf{e}_{j, k_{i}}^{\star}}{\prod_{k=1}^{n} \mathbf{e}_{j, k}^{\star}} \mathbf{e}_{j, k_{i}} .
$$

In above, for each $i$ we define $k_{i}$ so that $\mathbf{e}_{j, k_{i}}^{\star}\left(\mathbf{u}_{i}\right)=1$, i.e., $\mathbf{e}_{j, k_{i}}=\mathbf{u}_{i}$.

Lemma 9. The sum $\widetilde{\mathbf{e}}_{P}$ is equal to zero.

Proof. We can rewrite $\widetilde{\mathbf{e}}_{P}$ by

$$
\widetilde{\mathbf{e}}_{P}=\sum_{j=1}^{L} \frac{\mathbf{v}_{j}^{n-1}}{\prod_{k=1}^{n} \mathbf{e}_{j, k}^{\star}}\left(\sum_{k=1}^{n} \mathbf{e}_{j, k}^{\star} \mathbf{e}_{j, k}\right) .
$$

Since $\left\{\mathbf{e}_{j, k}^{\star}\right\}_{k}$ is the dual basis to $\left\{\mathbf{e}_{j, k}^{\star}\right\}_{k}$,

$$
\sum_{k=1}^{n} \mathbf{e}_{j, k}^{\star} \mathbf{e}_{j, k}
$$

is independent of $j$ as a polynomial on $N_{\mathbb{R}} \times M_{\mathbb{R}}$.

Hence, Proposition 3 implies

$$
\begin{aligned}
\widetilde{\mathbf{e}}_{P} & =\left(\sum_{k=1}^{n} \mathbf{e}_{j, k}^{\star} \mathbf{e}_{j, k}\right) \sum_{j=1}^{L} \frac{\mathbf{v}_{j}^{n-1}}{\prod_{k=1}^{n} \mathbf{e}_{j, k}^{\star}} \\
& =0
\end{aligned}
$$


as a polynomial, because

$$
\sum_{j=1}^{L} \frac{\mathbf{v}_{j}^{n-1}}{\prod_{k=1}^{n} \mathbf{e}_{j, k}^{\star}}=\left.\sum_{\sigma: \text { maximal }} e_{\sigma}\left(\sum_{i=1}^{K} \Psi_{i}\right)^{n-1}\right|_{\sigma}
$$

and the degree of the piecewise polynomial $\left(\sum_{i=1}^{K} \Psi_{i}\right)^{n-1}$ is strictly less than $n$. The proof is completed.

\section{Proof of Theorem 1}

\subsection{Strategy}

We will show Theorem 1 by proving that $\mathbf{e}_{P}$ is proportional to $\widetilde{\mathbf{e}}_{P}$ if $\mathbf{m}_{P^{*}}=\mathbf{o}$. Our strategy is as follows.

By the definition of $\widetilde{\mathbf{e}}_{P}$ and Proposition 6, $\widetilde{\mathbf{e}}_{P}$ is re-written as

$$
\widetilde{\mathbf{e}}_{P}=\sum_{i=1}^{K} A\left(F_{i}\right) \mathbf{u}_{i}
$$

In order to complete the proof of Theorem 1, it is sufficient to prove that

$$
A\left(F_{i}\right)=C \sharp \mathrm{V}\left(F_{i}\right)
$$

for some non-zero constant $C$ independent of $i$. By the definition of $\mathbf{m}\left(F_{i}\right)$, we have

$$
\mathbf{m}\left(F_{i}\right)\left(\mathbf{u}_{i}\right)=\left\langle\mathbf{u}_{i}, \mathbf{m}\left(F_{i}\right)\right\rangle=C \cdot A\left(F_{i}\right)
$$

for some non-zero constant $C$ independent of $i$. In the left hand of (14), we are substituting the vector $\mathbf{u}_{i}$ into $\mathbf{m}\left(F_{i}\right)$ as a polynomial.

The following equality describes the polar duality between $\mathbf{e}_{P}$ and $\mathbf{m}_{P^{*}}$ :

$$
\begin{aligned}
\sum_{i=1}^{K} \mathbf{u}_{i} \mathbf{m}\left(F_{i}\right) & =\sum_{i=1}^{K} \mathbf{u}_{i} \sum_{\mathbf{v}_{j} \in \mathrm{V}\left(F_{i}\right)} \frac{\mathbf{v}_{j}^{n} \mathbf{e}_{j, k_{i}}^{\star}}{\prod_{k=1}^{n} \mathbf{e}_{j, k}^{\star}} \\
& =\left(\sum_{j=1}^{L} \frac{\mathbf{v}_{j}^{n}}{\prod_{k=1}^{n} \mathbf{e}_{j, k}^{\star}}\right)\left(\sum_{k=1}^{n} \mathbf{e}_{j, k}^{\star} \mathbf{e}_{j, k}\right) .
\end{aligned}
$$

The left hand of (15) denotes the sum of the product the polynomial $\mathbf{u}_{i}$ on $M_{\mathbb{R}}$ and the polynomial $\mathbf{m}\left(F_{i}\right)$ on $N_{\mathbb{R}}$. On the other hand, we have

$$
\sum_{i=1}^{K} \sum_{\mathbf{v}_{j} \in \mathrm{V}\left(F_{i}\right)} \mathbf{u}_{i} \mathbf{v}_{j}=L\left(\sum_{k=1}^{n} \mathbf{e}_{j, k}^{\star} \mathbf{e}_{j, k}\right)
$$

Let us introduce the normalized relative momentum of $F_{i}$ by

$$
\widetilde{\mathbf{m}}\left(F_{i}\right):=\mathbf{m}\left(F_{i}\right)-\frac{1}{L}\left(\sum_{j=1}^{L} \frac{\mathbf{v}_{j}^{n}}{\prod_{k=1}^{n} \mathbf{e}_{j, k}^{\star}}\right) \sum_{\mathbf{v}_{j} \in \mathrm{V}\left(F_{i}\right)} \mathbf{v}_{j} .
$$

From (16) and (17), the equality (15) is equivalent to

$$
\sum_{i=1}^{K} \mathbf{u}_{i} \widetilde{\mathbf{m}}\left(F_{i}\right)=0 .
$$

From (14) and (17), the proof of Theorem 1 is reduced to prove

$$
\widetilde{\mathbf{m}}\left(F_{i}\right)\left(\mathbf{u}_{i}\right)=\left\langle\mathbf{u}_{i}, \widetilde{\mathbf{m}}\left(F_{i}\right)\right\rangle=0
$$


for each $i$. In fact, (19) implies that

$$
A\left(F_{i}\right)=\frac{1}{C L}\left(\sum_{j=1}^{L} \frac{\mathbf{v}_{j}^{n}}{\prod_{k=1}^{n} \mathbf{e}_{j, k}^{\star}}\right) \sharp \mathrm{V}\left(F_{i}\right)
$$

which is equivalent to (13).

\subsection{On the structure of $P^{\star}$ with $\mathbf{m}_{P^{\star}}=\mathbf{o}$}

In this subsection, we observe a combinatorial structure of $P^{\star}$ with $\mathbf{m}_{P^{\star}}=\mathbf{o}$. Through this subsection, we assume that $\mathbf{m}_{P^{\star}}=\mathbf{o}$.

Fix any facet $F$ of $P^{\star}$ which we regard as a bottom facet of $P^{\star}$. Let

$$
\begin{aligned}
\operatorname{RILP}(F) & :=\operatorname{Int}(F) \cap M \\
& =\left\{\mathbf{y} \in M \mid\left\langle\mathbf{u}_{F}, \mathbf{y}\right\rangle=-1,\left\langle\mathbf{u}_{G}, \mathbf{y}\right\rangle \geq 0, \forall G(\neq F) \text { facet }\right\}
\end{aligned}
$$

be the set of all relative interior lattice points in $F$. In above, $G$ runs through all facets of $P^{\star}$ except $F$.

\subsubsection{Case where $\operatorname{RILP}(F)$ is non-empty.}

We denote by

$$
\operatorname{RILP}(F)=\left\{\mathbf{y}_{1}, \cdots, \mathbf{y}_{k}\right\} .
$$

From Theorem 4.2 in [11], the condition $\mathbf{m}_{P^{\star}}=\mathbf{o}$ implies that the corresponding fan $\Sigma(P)$ is semisimple, i.e., for any relative interior lattice point $\mathbf{y}$ in any facet of $P^{\star}$, the antipodal point $-\mathbf{y}$ is also a relative interior lattice point in some facet. For any $\mathbf{y}_{i} \in \operatorname{RILP}(F)$, let $F_{\mathbf{y}_{i}}$ be the facet of $P^{\star}$ containing $-\mathbf{y}_{i}$ in its interior.

Lemma 10. The facet $F_{\mathbf{y}_{i}}$ does not contain the antipodal point of any other point in $\operatorname{RILP}(F)$ except $\mathbf{y}_{i}$ in its interior. That is to say,

$$
\left\langle\mathbf{u}_{\mathbf{y}_{i}},-\mathbf{y}_{j}\right\rangle \geq 0
$$

for any $j \neq i$, where $\mathbf{u}_{\mathbf{y}_{i}}$ is the normal vector of $F_{\mathbf{y}_{i}}$.

Proof. Assume that the facet $F_{\mathbf{y}_{i}}$ would contain another antipodal lattice point $-\mathbf{y}_{j}\left(\neq-\mathbf{y}_{i}\right)$ as its interior point. Let the lattice point $\mathbf{q}$ be $-\mathbf{y}_{i}+\mathbf{y}_{j}$. In addition, we can assume that $\mathbf{q}$ is the primitive vector without loss of generality (by dividing $\mathbf{q}$ by some integer if needed).

Take an arbitrary facet $G$ of $P^{\star}$ except $F$ or $F_{\mathbf{y}_{i}}$, and denote its normal vector by $\mathbf{u}_{G}$. Since $G$ is not equal to $F_{\mathbf{y}_{i}}$, the antipodal point $-\mathbf{y}_{i}$ was not contained in $G$. We had

$$
\left\langle\mathbf{u}_{G},-\mathbf{y}_{i}\right\rangle \geq 0 .
$$

Similarly, since $G$ is not equal to $F$, the lattice point $\mathbf{y}_{i}$ was not contained in $G$. We had

$$
\left\langle\mathbf{u}_{G}, \mathbf{y}_{j}\right\rangle \geq 0 \text {. }
$$

These two inequalities implied that

$$
\left\langle\mathbf{u}_{G}, \mathbf{q}\right\rangle=\left\langle\mathbf{u}_{G},-\mathbf{y}_{i}\right\rangle+\left\langle\mathbf{u}_{G}, \mathbf{y}_{j}\right\rangle \geq 0 .
$$

Since both of $\mathbf{y}_{j}$ and $\mathbf{y}_{i}$ were contained in $F$, we had

$$
\left\langle\mathbf{u}_{F}, \mathbf{y}_{i}\right\rangle=\left\langle\mathbf{u}_{F}, \mathbf{y}_{j}\right\rangle=-1
$$


where $\mathbf{u}_{F}$ is the normal vector of $F$. This implied

$$
\left\langle\mathbf{u}_{F}, \mathbf{q}\right\rangle=0 .
$$

Similarly, since both of $-\mathbf{y}_{j}$ and $-\mathbf{y}_{i}$ were contained in $F_{\mathbf{y}_{i}}$, we had

$$
\left\langle\mathbf{u}_{\mathbf{y}_{i}}, \mathbf{q}\right\rangle=0
$$

where $\mathbf{u}_{\mathbf{y}_{i}}$ is the normal vector of $F_{\mathbf{y}_{i}}$.

From (20), (21) and (22), we could conclude that

$$
\left\langle\mathbf{u}_{G}, \mathbf{q}\right\rangle \geq 0
$$

for all facets $G$ of $P^{\star}$. This implied that q was contained in the interior of $P^{\star}$ except the origin. This contradicts to that the fact that the origin is the only lattice point in the interior of $P^{\star}$. The proof is completed.

We call the facets $F_{\mathbf{y}_{i}}$ as above the top facets with respect to the bottom facet $F$.

Lemma 11. The convex hull $\operatorname{Conv}\left(\mathbf{o}, \mathbf{y}_{1}, \ldots, \mathbf{y}_{k}\right)$ is empty. Here we say that a lattice polytope is empty if it contains no lattice point except its vertices.

Proof. Assume that the convex hull $\operatorname{Conv}\left(\mathbf{0}, \mathbf{y}_{1}, \ldots, \mathbf{y}_{k}\right)$ would contain a lattice point $\mathbf{z}$ except its vertices. Since $\mathbf{z}$ was contained in the facet $F$, there existed non-negative constants $\gamma_{i}(1 \leq i \leq k)$ such that

$$
\mathbf{z}=\sum_{i=1}^{k} \gamma_{i} \mathbf{y}_{i}, \quad \sum_{i=1}^{k} \gamma_{i}=1, \quad \gamma_{i} \geq 0 .
$$

Since $\mathbf{z}$ was an relative interior lattice point of $F$, the semisimplicity of $\Sigma(P)$ implied that there existed a unique facet $F_{\mathbf{z}}$ containing $-\mathbf{z}$ in its interior. Let $\mathbf{u}_{\mathbf{z}}$ be the normal vector of $F_{\mathbf{z}}$. Since $F_{\mathbf{z}}$ did not contain any $-\mathbf{y}_{i}$ due to Lemma 10, for any $i$, we had

$$
\left\langle\mathbf{u}_{\mathbf{z}},-\mathbf{y}_{i}\right\rangle \geq 0 .
$$

Therefore, we had

$$
\left\langle\mathbf{u}_{\mathbf{z}},-\mathbf{z}\right\rangle=\sum_{i=1}^{k}\left\langle\mathbf{u}_{\mathbf{z}},-\gamma_{i} \mathbf{y}_{i}\right\rangle \geq 0 .
$$

On the other hand, we had

$$
\left\langle\mathbf{u}_{\mathbf{z}},-\mathbf{z}\right\rangle=-1,
$$

because $-\mathbf{z}$ was contained in $F_{\mathbf{z}}$, which is a contradiction.

Lemma 12. The convex hull $\operatorname{Conv}\left(\mathbf{0}, \mathbf{y}_{1}, \ldots, \mathbf{y}_{k}\right)$ is a $k$-simplex.

Proof. For any $i$, let us $\mathbf{u}_{\mathbf{y}_{i}}$ denote the normal vector of $F_{\mathbf{y}_{i}}$ as before. Let

$$
d=\operatorname{dim} \operatorname{Conv}\left(\mathbf{o}, \mathbf{y}_{1}, \ldots, \mathbf{y}_{k}\right) .
$$

Let us denote the hyperplane through the origin and parallel to $F_{\mathbf{y}_{i}}$ by

$$
H_{\mathbf{y}_{i}}=\left\{\mathbf{y} \in M_{\mathbb{R}} \mid\left\langle\mathbf{u}_{\mathbf{y}_{i}}, \mathbf{y}\right\rangle=0\right\} .
$$

From Lemma 10, we find that for each $\mathbf{y}_{i}$ there exists uniquely the top facet $F_{\mathbf{y}_{i}}$ such that

$$
\left\langle\mathbf{u}_{\mathbf{y}_{i}}, \mathbf{y}_{i}\right\rangle=-\left\langle\mathbf{u}_{\mathbf{y}_{i}},-\mathbf{y}_{i}\right\rangle=1 .
$$

For any other points $\mathbf{y}_{j}$ except $\mathbf{y}_{i}$, Lemma 11 induces that the lattice distance between $\mathbf{y}_{i}$ and $\mathbf{y}_{j}$ is equal to one. Then, we have

$$
\left\langle\mathbf{u}_{\mathbf{y}_{i}}, \mathbf{y}_{j}\right\rangle=\left\langle\mathbf{u}_{\mathbf{y}_{i}}, \mathbf{y}_{i}\right\rangle-1=0 .
$$


This means that for any $j$ except $i$, the vertex $\mathbf{y}_{j}$ is contained in the same hyperplane $H_{\mathbf{y}_{i}}$. Then, we find that the set

$$
\left\{\mathbf{y}_{i} \mid 1 \leq i \leq k\right\}
$$

of vectors in $M_{\mathbb{R}}$ are linearly independent. This means that the convex hull $\operatorname{Conv}\left(\mathbf{o}, \mathbf{y}_{1}, \ldots, \mathbf{y}_{k}\right)$ is a $k$ dimensional simplex. The proof is completed.

Lemma 13. Let $G$ be a facet of $P^{\star}$ except $F$, which does not contain any antipodal lattice point $-\mathbf{y}_{i}(1 \leq i \leq k)$. Let $\mathbf{u}_{G}$ be the normal vector of $G$. Then, the followings hold.

- For any $i(1 \leq i \leq k)$, we have

$$
\left\langle\mathbf{u}_{G}, \mathbf{y}_{i}\right\rangle=0 .
$$

- The facet $G$ is adjacent to $F$.

Proof. Since $-\mathbf{y}_{i}$ is not contained in $G$,

$$
\left\langle\mathbf{u}_{G},-\mathbf{y}_{i}\right\rangle \geq 0 .
$$

On the other hand, since $\mathbf{y}_{i}$ is not contained in $G$, we also have

$$
\left\langle\mathbf{u}_{G}, \mathbf{y}_{i}\right\rangle \geq 0 .
$$

These two inequalities imply that for any $i$,

$$
\left\langle\mathbf{u}_{G}, \mathbf{y}_{i}\right\rangle=0
$$

This is the first statement.

Let us show the second statement by contradiction. Assume that the facet $G$ would not be adjacent to $F$. Let

$$
H_{G}:=\left\{\mathbf{y} \in M_{\mathbb{R}} \mid\left\langle\mathbf{u}_{G}, \mathbf{y}\right\rangle=0\right\}
$$

be the hyperplane through the origin which is parallel to $G$. Remark that the lattice distance between $H_{G}$ and $G$ is equal to one by the definition of $P^{\star}$. Then, either $H_{G}$ did not intersect with $F$ or intersected with the boundary $\partial F$ of $F$, because $P^{\star}$ is convex. This implies that any $\mathbf{y}_{i}$ was not contained in $H_{G}$, because $\mathbf{y}_{i}$ is an interior point of $F$. This implied that for all $i(1 \leq i \leq k)$,

$$
\left\langle\mathbf{u}_{G}, \mathbf{y}_{i}\right\rangle>0 \text {. }
$$

This is a contradiction to the first statement of this lemma. Hence, the second statement is proved.

We call the facets $G$ as in Lemma 13 the side facets of $P^{\star}$ with respect to $F$.

Lemma 14. Any $k$-tuple of facets among

$$
\left\{F_{\mathbf{y}_{i}} \mid 1 \leq i \leq k\right\} \cup\{F\}
$$

has a non-empty intersection that is an ( $n-k)$-dimensional face of $P^{\star}$.

Proof. First, we will prove the statement for the intersection of $\left\{F_{\mathbf{y}_{i}} \mid 1 \leq i \leq k\right\}$. Let

$$
W_{F_{\mathbf{y}_{i}}}=\left\{\mathbf{y} \in M_{\mathbb{R}} \mid\left\langle\mathbf{u}_{\mathbf{y}_{i}}, \mathbf{y}\right\rangle=-1\right\}
$$

be the hyperplane containing the facet $F_{\mathbf{y}_{i}}$. Let

$$
Y=\cap_{i=1}^{k} W_{F_{\mathbf{y}_{i}}}
$$

be the intersection of the hyperplanes $W_{F_{\mathbf{y}_{i}}}$ for $1 \leq i \leq k$. Lemma 12 implies that the vectors $\mathbf{y}_{i}(1 \leq i \leq k)$ are $\mathbb{Z}$-linearly independent. Then, we find that the dimension of $Y$ is equal to $n-k$. 
Let us see that $Y$ contains an $(n-k)$-dimensional face of $P^{*}$ by contradiction. Assume that the affine space $Y$ would not contain any $(n-k)$-dimensional face of $P^{\star}$. Then, there existed a facet $G$ of $P^{\star}$ except $F_{\mathbf{y}_{i}}(1 \leq i \leq k)$ which has a non-empty intersection $Z$ with the cone

$$
\sum_{i=1}^{k} \mathbb{R}_{\geq 0} \cdot\left(-\mathbf{y}_{i}\right)
$$

Take a point $\mathbf{y}$ in $Z$ which is written by

$$
\mathbf{y}=\sum_{i=1}^{k} \gamma_{i}\left(-\mathbf{y}_{i}\right), \quad \gamma_{i} \geq 0 .
$$

Since any $-\mathbf{y}_{i}$ was not contained in $G$, the normal vector $\mathbf{u}_{G}$ of $G$ satisfied

$$
\left\langle\mathbf{u}_{G},-\mathbf{y}_{i}\right\rangle \geq 0
$$

for any $1 \leq i \leq k$. With this inequalities, the equality (23) implied that

$$
\left\langle\mathbf{u}_{G}, \mathbf{y}\right\rangle \geq 0 .
$$

This is a contradiction to that $\mathbf{y}$ is contained in $G$. Hence, we find that

$$
\cap_{1 \leq i \leq k} F_{\mathbf{y}_{i}}
$$

constitutes an $(n-k)$-dimensional face of $P^{\star}$. This is the statement what we desire to prove.

As for other intersections

$$
\left(\cap_{1 \leq j \neq i \leq k} F_{\mathbf{y}_{j}}\right) \cap F,
$$

the same argument can be applied by regrading the facet $F_{\mathbf{y}_{i}}$ as the bottom facet instead of $F$. The proof is completed.

Combining the results we proved so far, we can prove the following proposition.

Proposition 15. Let $P^{\star}$ be the polar dual polytope of a Fano polytope $P$ with $\mathbf{m}_{P^{\star}}=\mathbf{0}$. Assume that $P^{\star}$ admits a facet $F$ (the bottom) which contains relative interior lattice points $\left\{\mathbf{y}_{1}, \ldots, \mathbf{y}_{k}\right\}$ in $F$. For each $\mathbf{y}_{i}$, we denote by $F_{\mathbf{y}_{i}}$ the corresponding top facet as in Lemma 10 which contains $-\mathbf{y}_{i}$ as a relative interior lattice point. Then, the followings hold:

(i) Any side facet $G$ as in Lemma 13 is parallel to $\operatorname{Conv}\left(\mathbf{o}, \mathbf{y}_{1}, \ldots, \mathbf{y}_{k}\right)$. In particular, we have

$$
\left\langle\mathbf{u}_{G}, \sum_{i=1}^{k} \mathbf{y}_{i}\right\rangle=0
$$

where $\mathbf{u}_{G}$ is the normal vector of $G$.

(ii) The sum $\sum_{i=0}^{k} \widetilde{\mathbf{m}}\left(F_{\mathbf{y}_{i}}\right)$ of the normalized relative momentum of all top facets and the bottom facet $F$ of $P^{\star}$ is equal to the origin in $M_{\mathbb{R}}$, where $F_{\mathbf{y}_{0}}:=F$.

Proof. The statement (i) follows from Lemma 13 directly.

We will prove the condition (ii). From the statement (i) in this proposition, we find that any intersection of side facets has the dimension at most $k$. Lemma 14 implies that any $k$-tuple among the facets $\left\{F_{\mathbf{y}_{i}}\right\}_{0 \leq i \leq k}$ has the intersection of dimension $(n-k)$. Then, any vertex of $P^{\star}$ is the intersection of a $k$-tuple of the facets among $\left\{F_{\mathbf{y}_{i}}\right\}_{0 \leq i \leq k}$ and an $(n-k)$-tuple of the side facets, because the set of all facets of $P^{\star}$ consists of the bottom facet $F$, the top facets $\left\{F_{\mathbf{y}_{i}}\right\}_{1 \leq i \leq k}$ and the side facets. Conversely, the intersection of any $k$-tuple of the facets among $\left\{F_{\mathbf{y}_{i}}\right\}_{0 \leq i \leq k}$ and any $(n-k)$-tuple of the side facets constitutes a vertex of $P^{\star}$. The above observation implies the followings. 
- Any vertex $\mathbf{v}$ of any facet $F_{\mathbf{y}_{i}}$ is contained in the intersection of $F_{\mathbf{y}_{i}}$ and any $(k-1)$-tuple of the facets among $\left\{F_{\mathbf{y}_{j}}\right\}_{0 \leq j \leq k, j \neq i}$.

- The set of vertices of $\left\{F_{\mathbf{y}_{i}}\right\}_{0 \leq i \leq k}$ is equal to the set of vertices of $P^{\star}$.

These two facts imply

$$
\sum_{i=0}^{k} \sum_{\mathbf{v} \in \mathrm{V}\left(F_{\mathbf{y}_{i}}\right)} \mathbf{v}=k \sum_{\mathbf{v} \in \mathrm{V}\left(P^{*}\right)} \mathbf{v}=\mathbf{o} .
$$

The second equality in (25) follows from (3). The equality (25) implies that

$$
\sum_{i=0}^{k} \widetilde{\mathbf{m}}\left(F_{\mathbf{y}_{i}}\right)=\sum_{i=0}^{k} \mathbf{m}\left(F_{\mathbf{y}_{i}}\right) .
$$

We shall prove that the right hand in (26) is equal to zero. For a side facet $G$ with normal vector $\mathbf{u}_{G}$, we define the family of the hyperplanes $H_{G}(t)(0 \leq t \leq 1)$ parallel to $G$ by

$$
H_{G}(t):=\left\{\mathbf{y} \in M_{\mathbb{R}} \mid\left\langle\mathbf{u}_{G}, \mathbf{y}\right\rangle=-t\right\} .
$$

We define the vertical section of $P^{\star}$ cut by $H_{G}(t)$ for all side facets $G$ by

$$
S P^{\star}(t):=P^{\star} \cap\left(\cap_{G: \text { side }} H_{G}(t)\right) .
$$

By definition, $S P^{\star}(1)$ is equal to the union of all side facets.

Lemma 13 implies that $S P^{\star}(0)$ is equal to the $k$-dimensional simplex whose vertices are given by the average of all vertices of $F_{\mathbf{y}_{i}}(0 \leq i \leq k)$. The equality (25) implies that the sum of vertices of $S P^{\star}(0)$ is equal to zero. Since $S P^{\star}(0)$ is a simplex, we find that the momentum of $S P^{\star}(0)$ is also equal to zero.

Since any vertex of $P^{*}$ is the intersection of a $k$-tuple of the facets among $\left\{F_{\mathbf{y}_{i}}\right\}_{0 \leq i \leq k}$ and an $(n-k)$-tuple of the side facets, the intersection of $S P^{\star}(t)$ and any $F_{\mathbf{y}_{i}}$ is contained in the interior of $F_{\mathbf{y}_{i}}$ for $0 \leq t<1$ and $0 \leq i \leq k$. More precisely, the intersection

$$
\mathrm{SP}^{\star}(t) \cap F_{\mathbf{y}_{i}}
$$

deforms homothetically in $t$ and converges to a relative interior point in $F_{\mathbf{y}_{i}}$ as $t$ goes to zero. Moreover, a facet of $S P^{\star}(t)$ for $0<t \leq 1$ is homothetic to the one of side facets of $P^{\star}$ parallel to it. Therefore, we conclude that $P^{\star}$ can be divided into the union of $n$-dimensional pyramid which has $\operatorname{SP}^{\star}(0)$ as its apex and a side facet $G$ as its base. This implies that the momentum $\mathbf{m}\left(P^{\star}\right)$ is proportional to the sum of the momentum $\mathbf{m}(G)$ of all side facets $G$.

From the assumption that $\mathbf{m}\left(P^{\star}\right)$ is equal to zero, we have

$$
\sum_{G: \text { side }} \mathbf{m}(G)=\mathbf{o}
$$

Since

$$
\mathbf{m}\left(P^{\star}\right)=\sum_{G: \text { side }} \mathbf{m}(G)+\sum_{i=0}^{k} \mathbf{m}\left(F_{\mathbf{y}_{i}}\right)=\mathbf{o},
$$

we find that the right hand in (26) is equal to zero. The proof is completed.

\subsubsection{Case where $\operatorname{RILP}(F)$ is empty.}

Assume that a facet $F$ of $P^{\star}$ does not admit any relative interior lattice point. Let $\mathbf{u}_{F}$ be the normal vector of $F$. Since the fan $\Sigma(P)$ is complete, there exists a $k$-dimensional cone $\sigma$ such that $-\mathbf{u}_{F}$ is contained in the relative interior of $\sigma$. Let $\mathbf{u}_{F_{1}}, \cdots, \mathbf{u}_{F_{k}}$ be the set of 1-dimensional cones in $\Sigma(P)$ generating $\sigma$, where $F_{1}, \cdots, F_{k}$ are the corresponding facets respectively. 
Proposition 16. For the facets $\left\{F_{i} \mid 1 \leq i \leq k\right\}$, the equality

$$
\mathbf{u}_{F}+\sum_{i=1}^{k} \mathbf{u}_{F_{i}}=\mathbf{o}
$$

holds.

Proof. Let

$$
\mathbf{u}_{F}+\sum_{i=1}^{k} \alpha_{i} \mathbf{u}_{F_{i}}=\mathbf{o}, \alpha_{i} \in \mathbb{Z}_{>0} .
$$

If some $\alpha_{i}$ would be strictly larger than one, then the convex hull $\operatorname{Conv}\left(\mathbf{u}_{F}, \mathbf{u}_{F_{1}}, \cdots, \mathbf{u}_{F_{k}}\right)$ in $N_{\mathbb{R}}$ had at least one lattice point except the origin and its vertices. Since $\operatorname{Conv}\left(\mathbf{u}_{F}, \mathbf{u}_{F_{1}}, \cdots, \mathbf{u}_{F_{k}}\right)$ is contained in $P$, this is contradiction to that there is no lattice point on $P$ except the origin and its vertices.

Remark 17. This proposition holds whether $F_{i}$ admits a relative interior lattice point or not.

Proposition 18. Any facet $F_{i}(1 \leq i \leq k)$ as in Proposition 16 does not admit any relative interior lattice point.

Proof. The semisimplicity of $\Sigma(P)$ implies that any facet of $P^{\star}$ does not any relative interior lattice point $\mathbf{y}$ such that

$$
\left\langle\mathbf{u}_{F}, \mathbf{y}\right\rangle \geq 1 .
$$

In fact, if such $\mathbf{y}$ would be contained in some facet of $P^{\star}$, the semisimplicity of $\Sigma(P)$ implied that $-\mathbf{y}$ is also a relative interior lattice point of $F$. This is a contradiction to the assumption.

We prove the proposition by contradiction. Assume some $F_{i}$ would admit at least one relative interior lattice point. Then the inequality (28) implied that any relative interior lattice point $\mathbf{y}$ in $F_{i}$ satisfies

$$
\left\langle\mathbf{u}_{F}, \mathbf{y}\right\rangle=0 .
$$

Let $F_{\sigma}$ be the $(n-k)$-dimensional face of $P^{\star}$ corresponding to $\sigma$. Take a vertex $\mathbf{v}$ of $F_{\sigma}$. Let $\left\{\mathbf{e}_{j}^{\star}\right\}_{1 \leq j \leq n}$ be the set of the primitive vectors of the edges from $\mathbf{v}$ so that

$$
\left\langle\mathbf{u}_{F}, \mathbf{e}_{j}^{\star}\right\rangle \leq-1
$$

for $1 \leq j \leq k$ and

$$
\left\langle\mathbf{u}_{F}, \mathbf{e}_{j}^{\star}\right\rangle=0
$$

for the others. Remark that the edges $\mathbf{e}_{k+1}^{\star}, \ldots, \mathbf{e}_{n}^{\star}$ are contained in $F_{\sigma}$ and parallel to $F$. In addition, we assume that $\left\{\mathbf{e}_{j}^{\star}\right\}_{2 \leq j \leq k}$ is contained in $F_{i}$. Lemma 13 implies that the edge $\mathbf{e}_{1}^{\star}$, which is not contained in $F_{i}$, is parallel to the vector from the origin to some relative interior lattice point $\mathbf{y}^{\prime}$ in $F_{i}$. From (29), we have

$$
\left\langle\mathbf{u}_{F}, \mathbf{e}_{1}^{\star}\right\rangle=\left\langle\mathbf{u}_{F}, \mathbf{y}\right\rangle=0 .
$$

This is a contradiction to (30). Hence, any $F_{i}$ does admit no relative interior lattice point. The proof is completed.

Let $F_{1}, \cdots, F_{k}$ be the facets of $P^{\star}$ as in Proposition 18. Let $W$ be the $k$-dimensional subspace of $N_{\mathbb{R}}$ spanned by $\mathbf{u}_{F_{1}}, \cdots, \mathbf{u}_{F_{k}}$. Let us denote $F$ by $F_{0}$.

Proposition 19. Under the assumption $\mathbf{m}_{P^{*}}=\mathbf{0}$, the following properties concerning with $W$ and $\left\{F_{i}\right\}_{0 \leq i \leq k}$ hold.

(i) The vertices $\left\{\mathbf{u}_{F_{i}}\right\}_{0 \leq i \leq k}$ are the only vertices of $P$ contained in $W$.

(ii) The relative volume $A\left(F_{i}\right)$ of the facet $F_{i}$ is constant with respect to $0 \leq i \leq k$.

(iii) The number $\sharp \mathrm{V}\left(F_{i}\right)$ of the vertices of the facet $F_{i}$ is constant with respect to $0 \leq i \leq k$. 
Proof. Assume that the statement (i) would not hold. Then, there exists $\mathbf{u}^{\prime} \in \mathrm{V}(P) \cap W$ such that $\mathbf{u}^{\prime}$ is not equal to any $\mathbf{u}_{F_{i}}$ for $0 \leq i \leq k$. Since $\mathbf{u}_{F_{1}}, \cdots \mathbf{u}_{F_{k}}$ generates the cone $\sigma$, the vertex $\mathbf{u}^{\prime}$ existed outside of the cone $\sigma$. By (27), there existed an $l$-tuple $(1 \leq l \leq k)$ of vertices among $\left\{\mathbf{u}_{F_{i}}\right\}_{1 \leq i<k}$ (for simplicity, let denote them by $\mathbf{u}_{F_{1}}, \cdots, \mathbf{u}_{F_{l}}$ ) such that

$$
\mathbf{u}^{\prime}+\mathbf{u}_{F}+\sum_{j=1}^{l} \mathbf{u}_{F_{j}}=\mathbf{o} .
$$

From (27), we find that

$$
\mathbf{u}^{\prime}=\sum_{i=l+1}^{k} \mathbf{u}_{F_{j}},
$$

which is a contradiction to that $\mathbf{u}_{F_{1}}, \cdots \mathbf{u}_{F_{k}}$ generates the cone $\sigma$. Hence, the statement (i) is proved.

Next, we prove the statement (ii). Since $\mathbf{u}_{F_{i}}(0 \leq i \leq k)$ is contained the same hyperplane $W$, there exists an $(n-k)$-dimensional affine subspace $Z$ of $M_{\mathbb{R}}$ such that any facet $F_{i}(0 \leq i \leq k)$ is parallel to $Z$. Then, the polytope $P^{\star}$ can be divided into the union of the $(k+1)$-tuple of the pyramids whose apex is the intersection $P^{\star} \cap Z$, and whose base is $F_{i}(0 \leq i \leq k)$. From the statement (i) and (27), we find that if the relative volume $A\left(F_{i}\right)$ would be not constant in $i$, then the momentum of $P^{\star}$ sat outside of $Z$. In particular, the momentum of $P^{\star}$ was not equal to zero. This is a contradiction to the assumption. Hence, the statement (ii) is proved.

Finally, we prove the statement (iii). By the definition of $\sigma$ with respect to $F_{0}$ and the statement (i), we find that any $k$-tuple among $\left\{\mathbf{u}_{F_{0}}, \cdots, \mathbf{u}_{F_{k}}\right\}$ constitutes a $k$-dimensional cone of $\Sigma(P)$. Let $K_{i}$ be the $(n-k)$ dimensional face of $P^{\star}$ given by the intersection $\cap_{j \neq i} F_{j}$, and denote $N_{i}$ by the number of vertices of $K_{i}$. Since the polytope $P^{\star}$ can be divided into the union of the $(k+1)$-tuple of the pyramids whose apex is the intersection $P^{\star} \cap Z$, and whose base is $F_{i}(0 \leq i \leq k)$ as seen before, we find that any vertex of $P^{\star}$ is contained in either $K_{i}(0 \leq i \leq k)$ or the subspace $Z$. Assume that $N_{i}$ would be not constant in $i$. Then, we could assume that $N_{i_{0}}$ would is the maximum among $N_{0}, \cdots N_{k}$. From (27), then, we found that

$$
\sum_{\mathbf{v} \in \mathrm{V}\left(K_{i_{0}}\right)}\left\langle\mathbf{u}_{F_{i_{0}}}, \mathbf{v}\right\rangle=k N_{i_{0}},
$$

because the lattice distance between $\mathbf{v} \in \mathrm{V}\left(K_{i_{0}}\right)$ and $F$ is equal to $k+1$. For vertices of the other faces $K_{j}$ $\left(j \neq i_{0}\right)$, we had

$$
\sum_{\mathbf{v} \in \mathrm{V}\left(K_{j}\right)}\left\langle\mathbf{u}_{F_{i_{0}}}, \mathbf{v}\right\rangle=-N_{j},
$$

because the face $K$ is contained on $F_{i_{0}}$. Then, we had

$$
\begin{aligned}
\sum_{\mathbf{v} \in \mathrm{V}\left(P^{\star}\right)}\left\langle\mathbf{u}_{F_{i_{0}}}, \mathbf{v}\right\rangle & =\sum_{j \neq i_{0}} \sum_{\mathbf{v} \in \mathrm{V}\left(K_{j}\right)}\left\langle\mathbf{u}_{F_{i_{0}}}, \mathbf{v}\right\rangle+\sum_{\mathbf{v} \in \mathrm{V}\left(K_{i_{0}}\right)}\left\langle\mathbf{u}_{F_{i_{0}}}, \mathbf{v}\right\rangle+\sum_{\mathbf{v} \in \mathrm{V}\left(P^{*}\right) \cap Z}\left\langle\mathbf{u}_{F_{i_{0}}}, \mathbf{v}\right\rangle \\
& =-\sum_{j \neq i_{0}} N_{j}+k N_{i_{0}}>0 .
\end{aligned}
$$

This is a contradiction to the second equality in (25). Hence, the statement (iii) is proved.

\subsection{Completion}

In this subsection, we complete the proof of Theorem 1 by using the observations studied in the previous subsection. First, we will show the equalities (19) for facets admitting its relative interior lattice points.

Lemma 20. If a facet $F$ admits at least one relative interior lattice point, then the equalities (19) hold.

Proof. Assume that $F$ admits a $k$-tuple $\left\{\mathbf{y}_{1}, \ldots, \mathbf{y}_{k}\right\}$ of relative interior lattice points. Let

$$
\widetilde{\mathbf{y}}:=\frac{1}{k} \sum_{i=1}^{k} \mathbf{y}_{i} .
$$


To show a contradiction, we assume that

$$
\left\langle\mathbf{u}_{F}, \widetilde{\mathbf{m}}(F)\right\rangle<0
$$

without loss of generality. Then, Lemma 14 and Proposition 15 induce the followings.

1. There exist the corresponding top facets $F_{1}, \ldots, F_{k}$ to $\left\{\mathbf{y}_{1}, \ldots, \mathbf{y}_{k}\right\}$ such that

$$
\sum_{i=1}^{k}\left\langle\mathbf{u}_{F}, \widetilde{\mathbf{m}}\left(F_{i}\right)\right\rangle=-\left\langle\mathbf{u}_{F}, \widetilde{\mathbf{m}}(F)\right\rangle>0,
$$

2. $\left\langle\mathbf{u}_{F_{1}}, \widetilde{\mathbf{y}}\right\rangle=\cdots=\left\langle\mathbf{u}_{F_{k}}, \widetilde{\mathbf{y}}\right\rangle=\frac{1}{k}>0$,

3. $\left\langle\mathbf{u}_{F}, \widetilde{\mathbf{y}}\right\rangle=-1<0$, and

4. $\left\langle\mathbf{u}_{G}, \widetilde{\mathbf{y}}\right\rangle=0$ for any side facet $G$.

Remark that the last statement follows from that $G$ is parallel to $\operatorname{Conv}\left(\mathbf{o}, \mathbf{y}_{1}, \ldots, \mathbf{y}_{k}\right)$.

Substituting $\widetilde{\mathbf{y}}\left(\operatorname{resp} . \mathbf{u}_{F}\right)$ into $\mathbf{u}_{F_{i}}\left(\operatorname{resp} . \widetilde{\mathbf{m}}\left(F_{i}\right)\right)$ as a monomial on $N_{\mathbb{R}}\left(\operatorname{resp} . N_{\mathbb{R}}\right)$, we have

$$
\begin{aligned}
\mathbf{u}_{F}(\widetilde{\mathbf{y}}) \cdot \widetilde{\mathbf{m}}(F)\left(\mathbf{u}_{F}\right)+\sum_{i=1}^{k} \mathbf{u}_{F_{i}}(\widetilde{\mathbf{y}}) \cdot \widetilde{\mathbf{m}}\left(F_{i}\right)\left(\mathbf{u}_{F}\right) & =-\widetilde{\mathbf{m}}(F)\left(\mathbf{u}_{F}\right)+\frac{1}{k} \sum_{i=1}^{k} \widetilde{\mathbf{m}}\left(F_{i}\right)\left(\mathbf{u}_{F}\right) \\
& =-\left\langle\mathbf{u}_{F}, \widetilde{\mathbf{m}}(F)\right\rangle+\frac{1}{k} \sum_{i=1}^{k}\left\langle\mathbf{u}_{F}, \widetilde{\mathbf{m}}\left(F_{i}\right)\right\rangle \\
& >0 .
\end{aligned}
$$

However, the above inequality is a contradiction to (18). Hence we have

$$
\widetilde{\mathbf{m}}(F)\left(\mathbf{u}_{F}\right)=\left\langle\mathbf{u}_{F}, \widetilde{\mathbf{m}}(F)\right\rangle=0 .
$$

The proof is completed.

Lemma 20 implies that there exists some positive constant $C$ independent of $i$ satisfying

$$
\mathbf{e}_{P}=C \sum_{F} A(F) \mathbf{u}_{F}+\sum_{F^{\prime}} \sharp \mathrm{V}\left(F^{\prime}\right) \mathbf{u}_{F^{\prime}}
$$

where $F$ runs through the set of facets with at least one relative interior lattice points in the first summation, and $F^{\prime}$ runs through the set of facets without relative interior lattice points in the second summation.

Let us consider the second summation in the right hand of (31). Proposition 16 and Proposition 19 imply that the set of facets without relative interior lattice points is the disjoint union of tuples of the facets

$$
\bigsqcup_{j}\left\{F_{0, j}, \cdots, F_{k_{j}, j}\right\}, \quad \operatorname{RILP}\left(F_{i, j}\right)=\emptyset
$$

such that for each $j$

- the vectors $\mathbf{u}_{F_{0, j}}, \cdots \mathbf{u}_{F_{k_{j}, j}}$ span an affine subspace of $N_{\mathbb{R}}$,

- the summation $\mathbf{u}_{F_{0, j}}+\cdots+\mathbf{u}_{F_{k_{j}, j}}$ of the normal vectors is equal to $\mathbf{o}$,

- $A\left(F_{0, j}\right)=\cdots=A\left(F_{k_{j}, j}\right)$, and

- $\sharp \mathrm{V}\left(F_{0, j}\right)=\cdots=\sharp \mathrm{V}\left(F_{k, j}\right)$.

Then, for each $j$, we have

$$
\sharp \mathrm{V}\left(F_{0, j}\right) \mathbf{u}_{F_{0, j}} \cdots+\sharp \mathrm{V}\left(F_{k_{j}, j}\right) \mathbf{u}_{F_{k_{j}, j}}=A\left(F_{0, j}\right) \mathbf{u}_{F_{0, j}}+\cdots+A\left(F_{k_{j}, j}\right) \mathbf{u}_{F_{k_{j}, j}}=\mathbf{o} .
$$

Substituting the above into (31), we have

$$
\mathbf{e}_{P}=C \sum_{F: \text { facet of } P^{\star}} A(F) \mathbf{u}_{F}=C \widetilde{\mathbf{e}}_{P}=\mathbf{o} .
$$

The proof of Theorem 1 is completed. 


\section{Counterexamples to the converse of Theorem 1}

In this section, we show counterexamples to the converse of Theorem 1. We find such examples among the database of Fano polytopes given by Øbro [12] and Paffenholz [14] by using Polymake [7]. Up to the dimension 5, the converse of Theorem 1 holds. Among 6-dimensional Fano polytopes, there exist just two examples where $\mathbf{e}_{P}=\mathbf{o}$ but $\mathbf{m}_{P^{\star}} \neq \mathbf{o}$. The vertices of such examples $P$ are given as follows.

$$
\begin{aligned}
& \left(\begin{array}{ccccccccc}
0 & 0 & 0 & 0 & 0 & -1 & 1 & 0 & 0 \\
0 & 0 & -1 & 0 & 0 & 0 & 0 & 0 & 1 \\
-1 & 0 & 0 & 0 & 0 & 0 & 0 & 1 & 0 \\
0 & -1 & 0 & 0 & 0 & 0 & 1 & 0 & 0 \\
0 & 0 & 0 & -1 & 0 & 0 & 1 & 1 & -1 \\
0 & 0 & 0 & 0 & -1 & 0 & 0 & 0 & 1
\end{array}\right) \\
& \mathbf{5 9 6 3}\left(\begin{array}{cccccccccccc}
0 & 0 & 0 & 0 & 0 & 0 & 0 & 0 & 0 & 0 & -1 & 1 \\
0 & -1 & 0 & 1 & 0 & 0 & 0 & 0 & 0 & 0 & 0 & 0 \\
1 & 0 & 0 & 0 & 0 & -1 & 0 & 0 & 0 & 0 & 0 & 0 \\
1 & 0 & 0 & -1 & 0 & 0 & 0 & 0 & -1 & 1 & 0 & 0 \\
0 & 0 & 0 & 0 & 0 & 0 & 1 & -1 & 0 & 0 & 0 & 0 \\
-1 & 0 & -1 & 0 & 1 & 0 & 1 & 0 & 0 & -1 & 0 & 1
\end{array}\right)
\end{aligned}
$$

In above, the numbers denoted in boldface denote the indices of polytopes in the database (fano-v6d.tgz)[14]. The following table (Table 1) indicates the numbers of polytopes with $\mathbf{m}_{P^{\star}}=\mathbf{o}$ (resp. $\mathbf{e}_{P}=\mathbf{o}$ ) among Fano polytopes up to the dimension 7 .

Table 1: The number of Fano polytopes

\begin{tabular}{|l|c|c|c|c|c|c|c}
\hline dimension & 1 & 2 & 3 & 4 & 5 & 6 & 7 \\
\hline Fano polytopes & 1 & 5 & 18 & 124 & 866 & 7622 & 72256 \\
\hline $\mathbf{m}_{P^{\star}}=0$ & 1 & 3 & 5 & 12 & 23 & 51 & 100 \\
\hline $\mathbf{e}_{P}=0$ & 1 & 3 & 5 & 12 & 23 & 53 & 106 \\
\hline
\end{tabular}

Acknowledgement: This work was supported by JSPS KAKENHI Grant Number 17 K05233.

Conflict of interest: Authors state no conflict of interest.

\section{References}

[1] V.V. Batyrev and E.N. Selivanova, "Einstein-Kähler metrics on symmetric toric Fano manifolds" J. Reine Angew. Math. 512 (1999)225-236.

[2] M. Beck and S. Robins, "Computing the Continuous Discretely", UTM, Springer-Verlag, New York, 2007.

[3] M. Brion, "Points entiers dans les polyèdres convexes", Ann. Sci. École Norm. Sup. 21 (1988), no.4, 653-663.

[4] C. Casagrande, "The number of vertices of a Fano polytope”, Ann. Inst. Fourier (Grenoble) 56 (2006), no. 1, 121-130.

[5] A. Futaki, “An obstruction to the existence of Einstein Kähler metrics”, Invent. Math. 73, no. 3 (1983) 437-443.

[6] A. Futaki and T. Mabuchi, "Bilinear forms and extremal Kähler vectorfelds associated with Kähler classes”, Math. Ann. 301, no.2 (1995) 199-210.

[7] M. Joswig, B. Müller and A. Paffenholz, Polymake and lattice polytopes. In C. Krattenthaler, V. Srehl and M. Kauers, editors, DMTCS Proceedings of FPSAC, 2009, 491-502.

[8] E. Katz and S. Payne, "Piecewise polynomials, Minkowski weights, and localization on toric varieties", Algebra Number Theory 2 (2008), no. 2, 135-155. 
[9] T. Mabuchi, “Einstein-Kähler forms, Futaki invariants and convex geometry on toric Fano varieties”, Osaka J. Math. 24, no.4 (1987) 705-737.

[10] Y. Nakagawa, "Combinatorial formulae for Futaki characters and generalized Killing forms of toric Fano orbifolds", The Third Pacific Rim Geometry Conference (Seoul, 1996), 223-260, Monogr. Geom. Topology, 25, Int. Press, Cambridge, MA, 1998.

[11] B. Nill, “Complete toric varieties with reductive automorphism group” Math. Z. 252 (2006), no. 4, 767-786.

[12] M. Øbro, “Classification of smooth Fano polytopes”, Ph.D. thesis, University of Aarhus (2007). available at http://pure.au. $\mathrm{dk} /$ portal/files/41742384/imf_phd_2008_moe.pdf

[13] B. Nill and A. Paffenholz, "Examples of Kähler-Einstein toric Fano manifolds associated to non-symmetric reflexive polytopes”, Beitr. Algebra Geom. 52(2), 297-304 (2011).

[14] A. Paffenholz, "Smooth Reflexive Lattice Polytopes" at https://polymake.org/polytopes/paffenholz/www/fano.html.

[15] G. Tian and X.,Zhu, "A new holomorphic invariant and uniqueness of Kähler-Ricci solitons.", Comment. Math. Helv. 77, no.2 (2002) 297-325.

[16] X.J. Wang and X. Zhu, “Kähler-Ricci solitons on toric manifolds with positive first Chern class”, Adv. Math. 188, no.1 (2004) 87-103. 